\title{
Tumor mutational profile of triple negative breast cancer patients in Thailand revealed distinctive genetic alteration in chromatin remodeling gene
}

\author{
Suvimol Niyomnaitham ${ }^{1}$, Napa Parinyanitikul ${ }^{2}$, Ekkapong Roothumnong ${ }^{3,4}$, Worapoj Jinda ${ }^{4,5}$, Norasate \\ Samarnthai ${ }^{6}$, Taywin Atikankul ${ }^{7}$, Bhoom Suktitipat ${ }^{4,8,9}$, Wanna Thongnoppakhun ${ }^{4,5}$, Chanin Limwongse ${ }^{3,4,5}$ \\ , Manop Pithukpakorn Corresp. 3,4 \\ 1 Department of Pharmacology, Faculty of Medicine Siriraj Hospital, Mahidol University, Bangkok, Thailand \\ 2 Department of Medicine, Faculty of Medicine, Chulalongkorn University, Bangkok, Thailand \\ 3 Department of Medicine, Faculty of Medicine Siriraj Hospital, Mahidol University, Bangkok, Thailand \\ 4 Siriraj Center of Research Excellence in Precision Medicine, Faculty of Medicine Siriraj Hospital, Mahidol University, Bangkok, Thailand \\ 5 Research Division, Faculty of Medicine Siriraj Hospital, Mahidol University, Bangkok, Thailand \\ 6 Department of Pathology, Faculty of Medicine Siriraj Hospital, Mahidol University, Bangkok, Thailand \\ 7 Department of Pathology, Queen Savang Vadhana Memorial Hospital, Thai Red Cross Society, Chonburi, Thailand \\ 8 Department of Biochemistry, Faculty of Medicine Siriraj Hospital, Mahidol University, Bangkok, Thailand \\ 9 Integrative Computational Bioscience Center, Mahidol University, Bangkok, Thailand \\ Corresponding Author: Manop Pithukpakorn \\ Email address: manop.pit@mahidol.ac.th
}

Background. Triple negative breast cancer (TNBC) is a breast cancer subtype characterized by absence of both hormonal receptors and human epithelial growth factor receptor 2 (HER2). TNBC accounts for 15$20 \%$ of breast cancer. TNBC is associated with more aggressive disease and worse clinical outcome. Though the underlying mechanism of TNBC is currently unclear, the heterogeneity of clinical characteristics in various population may relate to the difference in tumor mutational profile. There were studies on TNBC gene mutations in various ethnic groups but the tumor genome data on Thai TNBC patients is currently unknown. This study aims to investigate mutational profile of Thai TNBC.

Methods. The patients were Thai individuals who were diagnosed with primary breast carcinoma between 2014 and 2017. All surgically removed primary tumor tissues were carefully examined by pathologists and archived as formalin-fixed paraffin embedded tumor. TNBC was defined by absence of hormonal receptors and HER2 by immunohistochemistry. Genomic DNA (gDNA) was extracted, enriched, and sequenced of all exomes on the Illumina HiSeq. Genomic data were then processed through bioinformatics platform to identify genomic alterations and tumor mutational burden.

Results. A total of 116 TNBC patients were recruited. Genomic analysis of TNBC samples identified 81,460 variants, of which 5,906 variants were in cancer associated genes. The result showed that Thai TNBC has higher tumor mutation burden than previously reported data. The most frequently mutated cancer-associated gene was TP53 similar to other TNBC cohorts. Meanwhile KMT2C was found to be more commonly mutated in Thai TNBC than previous studies. Mutational profile of Thai TNBC patients also revealed difference in many frequently mutated genes when compare to other Western TNBC cohorts.

Conclusion. This result supported that TNBC breast cancer patients from various ethnic background showed diverse genome alteration pattern. Although TP53 is the most commonly mutated gene across all cohorts, Thai TNBC showed different gene mutation frequencies, especially in KMT2C. In particular, the cancer gene mutations are more prevalent in Thai TNBC patients. This result provides important insight 
on diverse underlying genetic and epigenetic mechanisms of TNBC that could translate to a new treatment strategy for patients with this disease. 
1 Tumor Mutational Profile of Triple Negative Breast Cancer Patients in Thailand Revealed

\section{Distinctive Genetic Alteration in Chromatin Remodeling Gene}

3 Suvimol Niyomnaitham ${ }^{1}$, Napa Parinyanitikul ${ }^{2}$, Ekkapong Roothumnong ${ }^{3,4}$, Worapoj Jinda ${ }^{4,5}$,

4 Norasate Samarnthai $^{6}$, Taywin Atikankul ${ }^{7}$, Bhoom Suktitipat ${ }^{4,8,9}$, Wanna Thongnoppakhun ${ }^{4,5}$,

5 Chanin Limwongse $3,4,5$, Manop Pithukpakorn $3,4 *$

6 Department of Pharmacology, Faculty of Medicine Siriraj Hospital, Mahidol University,

7 Bangkok, Thailand

$8 \quad{ }^{2}$ Department of Medicine, Faculty of Medicine, Chulalongkorn University, Bangkok, Thailand

$9{ }^{3}$ Department of Medicine, Faculty of Medicine Siriraj Hospital, Mahidol University, Bangkok,

10 Thailand

$11{ }^{4}$ Siriraj Center of Research Excellence in Precision Medicine, Faculty of Medicine Siriraj

12 Hospital, Mahidol University, Bangkok, Thailand

$13{ }^{5}$ Research Division, Faculty of Medicine Siriraj Hospital, Mahidol University, Bangkok,

14 Thailand

${ }^{6}$ Department of Pathology, Faculty of Medicine Siriraj Hospital, Mahidol University, Bangkok,

16 Thailand

$17{ }^{7}$ Department of Pathology, Queen Savang Vadhana Memorial Hospital, Thai Red Cross Society,

18 Chonburi, Thailand

$19{ }^{8}$ Department of Biochemistry, Faculty of Medicine Siriraj Hospital, Mahidol University,

20 Bangkok, Thailand

$21{ }^{9}$ Integrative Computational Bioscience Center, Mahidol University, Bangkok, Thailand 
$22 *$ Corresponding Author

23 Manop Pithukpakorn

24 Department of Medicine

25 Faculty of Medicine Siriraj Hospital, Mahidol University

262 Wang Lang Road, Bangkoknoi

27 Bangkok, THAILAND 10700

28 Tel: 66-2-419-2727

29 Email: manop.pit@mahidol.ac.th

30 Running Title: Tumor Mutational Profile of Thai Triple Negative Breast Cancer

31 Keyword: Breast, Cancer, Thai, Asian, Genome

32 Total Word Count: 4,241

33 Abstract Word Count: 350

34

35

36

37

38

39 
40 Abstract

41 Background. Triple negative breast cancer (TNBC) is a breast cancer subtype characterized by

42 absence of both hormonal receptors and human epithelial growth factor receptor 2 (HER2).

43 TNBC accounts for $15-20 \%$ of breast cancer. TNBC is associated with more aggressive disease

44 and worse clinical outcome. Though the underlying mechanism of TNBC is currently unclear,

45 the heterogeneity of clinical characteristics in various population may relate to the difference in

46 tumor mutational profile. There were studies on TNBC gene mutations in various ethnic groups

47 but the tumor genome data on Thai TNBC patients is currently unknown. This study aims to

48 investigate mutational profile of Thai TNBC.

49 Methods. The patients were Thai individuals who were diagnosed with primary breast carcinoma between 2014 and 2017. All surgically removed primary tumor tissues were carefully

51 examined by pathologists and archived as formalin-fixed paraffin embedded tumor. TNBC was

52 defined by absence of hormonal receptors and HER2 by immunohistochemistry. Genomic DNA

53 (gDNA) was extracted, enriched, and sequenced of all exomes on the Illumina HiSeq. Genomic

54 data were then processed through bioinformatics platform to identify genomic alterations and

55 tumor mutational burden.

56 Results. A total of 116 TNBC patients were recruited. Genomic analysis of TNBC samples

57 identified 81,460 variants, of which 5,906 variants were in cancer associated genes. The result 58 showed that Thai TNBC has higher tumor mutation burden than previously reported data. The most frequently mutated cancer-associated gene was TP53 similar to other TNBC cohorts.

60 Meanwhile $K M T 2 C$ was found to be more commonly mutated in Thai TNBC than previous

61 studies. Mutational profile of Thai TNBC patients also revealed difference in many frequently

62 mutated genes when compare to other Western TNBC cohorts. 
63 Conclusion. This result supported that TNBC breast cancer patients from various ethnic

64 background showed diverse genome alteration pattern. Although TP53 is the most commonly

65 mutated gene across all cohorts, Thai TNBC showed different gene mutation frequencies,

66 especially in $K M T 2 C$. In particular, the cancer gene mutations are more prevalent in Thai TNBC

67 patients. This result provides important insight on diverse underlying genetic and epigenetic

68 mechanisms of TNBC that could translate to a new treatment strategy for patients with this

69 disease. 
83

84

\section{Introduction}

Triple negative breast cancer (TNBC) accounts for approximately $15-20 \%$ of breast cancer (Blows et al. 2010). Breast cancer patients with TNBC are not eligible for effective selective hormonal modulator or anti-HER 2 treatments because of the absence of both hormonal and growth factor receptor overexpression. Chemotherapy was therefore the only available treatment of patients with TNBC. Women with TNBC displayed a clinical aggressiveness and high risk of metastasis. TNBC has also been shown to associated with the poorer prognosis and reduced 5year survival than other breast cancer subtypes (Malorni et al. 2012). Several studies showed the substantial racial variations of clinical behavior and prevalence of TNBC, likely owing to a heterogeneous nature of the disease. African descents are more often to present with TNBC, higher histologic grade and more aggressive breast tumors than whites (Chen \& Li 2015). Both Hispanic and African women tend to be diagnosed in more advanced stage (Banegas \& Li 2012). Studies in Asian population demonstrated that $11 \%$ of breast cancer patients in Singapore was TNBC while this subtype accounted for $19 \%$ of Korean breast cancer patients (Rhee et al. 2008; Thike et al. 2009). The heterogeneity of TNBC on the clinical presentation as well as histologic differences may relate to variation in genetic background. The genomic profiles of TNBC in African Americans patients have been studied (Ademuyiwa et al. 2017; Huo et al. 2017). This study addressed the lack of data on the genomic profiles of TNBC in Thai and Asian population and investigated racial differences in the genetic landscape of breast cancer that could potentially identify targets suitable for specific population. 


\section{Methods}

107

108

109

110

111

112

113

114

115

116

117

118

119

120

121

122

123

124

125

126

127

\section{Study Population}

The study protocol was approved by the Siriraj and King Chulalongkorn Memorial Hospital Institutional Review Boards (Protocol No. 175/2559 and 642/2557). The study was conducted according to the Good Clinical Practice and the Declaration of Helsinki. All participates provided written informed consent. One hundred and sixteen Thai patients who were diagnosed with primary TNBC and treated at both hospitals between 2014 and 2017 were included. All 116 patients were female. The average age at diagnosis was $56.47 \pm 11.90$ years $( \pm \mathrm{SD})$ with an age range between 25 and 79 years. The BMI was $26.28 \pm 5.89 \mathrm{~kg} / \mathrm{m} 2$. Majority of the patients (87\%) were categorized as early stage breast cancer (20\% as stage I and $67 \%$ as stage IIa). Fiftyfive patients had follow-up period up to 3 years. There were 6 clinical relapses with in the 3 years follow-up period; 4 cases were in stage IIa and 2 cases were in stage IV. One patient died during follow-up period whose cause of death did not appear to be cancer-related.

Primary tumor tissues and lymph nodes were surgically removed as a standard treatment at Siriraj and King Chulalongkorn Memorial Hospitals and examined by board-certified pathologists. The tissues were dissected for histological diagnosis and immunohistochemistry staining then archived as FFPE tumor block. TNBC subtype was defined by absence of estrogen receptor (ER), progesterone receptor $(\mathrm{PR})$ and HER2 by immunohistochemistry staining with appropriate positive control. No amplification of HER2 was also confirmed by fluorescence insitu hybridization (FISH). Independent pathologists determined only the samples of primary TNBC tumors, which had more than 50\% tumor content after dissection, to be analyzed in this study. The FFPE primary tumors were sectioned into 10 micrometers using a new blade and 
128 preserved in 1.5ml Eppendorf tubes. Blade was changed for every tissue block to prevent the 129 contamination of DNA.

130 Tumor Genome Sequencing and Variant Calls

131 The genomic DNA (gDNA) was extracted using Qiagen DNeasy DNA Isolation Kit (Hilden,

132 Germany). FFPE gDNA (50-150 ng) was converted into libraries and enriched for whole exome 133 sequencing using Agilent's SureSelect Human All Exon V5 + UTR Sample Prep kit. Sequencing 134 was performed on the Illumina HiSeq 2500/4000 platform with average 300x sequencing depth. 135 Genomic data were then processed through bioinformatics platform and knowledge base (Wuxi 136 NextCODE Genomics, Boston, MA, USA) to identify genomic alterations including single 137 nucleotide polymorphisms/substitutions (SNPs) and small insertions/deletions (indels). A 138 threshold of 5\% allelic fraction was used for SNPs and indels. Any variants presented at allele 139 frequency above $1 \%$ in dbSNP, 1000 Genomes and ExAC databases were removed. To assess 140 somatic status of mutations in a tumor-only setting, we used both MuTect2 (Broad Institute, 141 Boston, MA, USA) and VarScan2 (Washington University, St. Louis, MO, USA) on the aligned 142 sequence data to determine somatic variants. All variants are further annotated with the extensive 143 pipeline including COSMIC data annotations. Additional annotation includes HGMD

144 Professional, ClinVar, OMIM, and multiple missense functional predictors including polyphen2, 145 SIFT, LRT, MutationTaster, MutationAssessor, and CADD. The effect of the sequence variants 146 on all protein coding genes in the RefSeq database was further annotated using the Variant Effect 147 Predictor, which predicts the consequence of each sequence variant on all neighboring RefSeq 148 genes based on a set of 35 consequence terms defined by the Sequence Ontology (SO) (McLaren 149 et al. 2016). Only variants predicted to cause strong and moderate alteration on gene functions, 150 such as stop gained/lost variants, frameshift, indels, donor/acceptor splice variants, initiator 
151 codon variants, missense variants, in-frame indels and splice region variants were selected for 152 analysis. Finally, the results were manually reviewed by molecular geneticists. These analysis 153 methods applied to the TCGA tumor variants and expression data have distinguished different 154 molecular or histologic subtypes of breast cancer at over 97\% accuracy.

\section{Data Comparison with Other Breast Cancer Cohorts}

156 To compare frequency of cancer gene alterations between this study and previously published 157 data, breast cancer mutation data from The Cancer Genome Atlas (TCGA), METABRIC and 158 French cohorts were downloaded from the cBioPortal for Cancer Genomics

159 (http://www.cbioportal.org). TCGA cohort consisted of cancer genome data form primary breast

160

161

162

163

164

165

166

167

168

169

170

171

172 cancer patients in the United States (Network 2012). METABRIC cohort data was collected from primary breast cancer patients in the United Kingdom and Canada (Pereira et al. 2016). French cohort data was primarily from the patients with metastatic breast cancers from 4 different prospective trials in France (Lefebvre et al. 2016). Cancer gene mutation data from TCGA database were selected only from breast invasive ductal carcinoma that was classified as PAM50 basal subtype, which is closely related to triple negative subtype of breast cancer, while data from METABRIC and French cohorts were selected from samples with negative ER, PR and HER2, similar to this study. The comparison of mutation frequency of each cancer genes was trimmed to 173 genes due to data limitation from 173-gene panel in METABRIC cohort.

\section{Statistical Analysis and Data Visualization}

Genomic characteristics were compared across cohorts using one-way analysis of variance for continuous variables. The prevalence of somatic mutations was compared across cohorts.

Descriptive statistics was used to show the average age and BMI of population. Statistical 
173 analyses were conducted using SAS software (version 9.4, SAS Institute, Cary, NC). A two-

174 tailed p-value less than 0.05 was considered significant. Landscape of co-occurrence and mutual

175 exclusion of cancer gene mutations was generated with OncoPrinter (version 1.0.1, cBioPortal

176 for Cancer Genomics, New York, NY). Mutational spectrum of 3 most commonly mutated genes

177 in lollipop plot format was generated with MutationMapper (version 1.0.1, cBioPortal for Cancer

178 Genomics, New York, NY) (Gao et al. 2013).

\section{Results}

\section{Comparison of Gene Alterations between Thai TNBC and Other TNBC Cohorts}

181

182

183

184

185

186

187

188

189

190

191

192

193

194

A total of 1,088,237 variants were detected from the whole exome sequencing data of 116 patients. Variant filtering with MuTect2 and VarScan2 identified 81,460 somatic variants that passed data analysis algorithms. When all 969 known or potential cancer-associated genes were examined, only 5,906 somatic variants were identified. We found an average of 222 variants per sample (range 104-388 variants) with an average of 20 altered cancer genes (range 5-35 genes). The 10 most commonly mutated genes were TP53, KMT2C, SYNE1, PIK3CA, BRCA1, NF1, BRCA2, PTEN, RB1 and ARIDIB (Figure 1 and Table 1 and 2). Comparing to the cancer gene mutation frequencies from TCGA, METABRIC and French cohorts, Thai TNBC had significantly higher mutation frequencies in KMT2C, SYNE1, PIK3CA, NF1, PTEN, BRCA1 and $B R C A 2$. On the contrary, significant differences were observed only in SYNE1 and BRCA1 among TCGA, METABRIC and French cohorts.

\section{Driver Gene Analysis}

We performed analysis of the filtered cancer genes to identify potential genes of interest. We first examined for genes mutated in multiple samples and found that 14 genes were mutated in at 
195 least $10 \%$ of the samples. SYNE1 was excluded from analysis due to unclear role in cancer. TP53

196 was the most frequently mutated gene with variants found in 88 samples. KMT2C, which

197 encodes for the lysine methyltransferase MLL3, was the next most frequently mutated gene with

198 mutations in 67 samples. The third most frequently mutated gene was $P I K 3 C A$, which was

199 shared by 27 samples. Together, 111 of the 116 samples had an alteration in at least TP53,

200 KMT2C, or PIK3CA (Figure 2). The most commonly recurring mutation was the PIK3CA

201 H1074R mutation, which was found in the majority of the PIK3CA mutant samples (16 out of 27

202 samples; 59\%). Within these genes, we also found a number of recurrent variants including the

203 previously reported mutations in KMT2C S338L (21 samples; 31\%), TP53 R175H (10 samples;

204 11\%), R248Q (5 samples; 6\%), Y220C (4 samples; 5\%), and R273H (4 samples; 5\%) and

205 PIK3CA K733R (6 samples; 22\%) and E545K (4 samples; 15\%) (Figure 3)

TP53 Mutation Status and Co-occurring Mutations

207

208

209

210

211

212

213

214

215

216

217

As previously shown, TP53 was the most commonly mutated gene in the cohort with 88 of the 116 samples $(76 \%)$ containing a mutation. To identify roles of TP53 and its association with the other 2 most commonly mutated genes; KMT2C and PIK3CA, we subdivided the cohort as TP53 wild-type and TP53 mutant groups. In the TP53 wild-type samples ( $\mathrm{n}=28)$, the PI3K pathway appeared to be a predominant driver with 19 samples $(68 \%)$ containing a mutation in either the PIK3CA gene or PI3K pathway members, including PIK3C2B, PIK3CG, and PTEN. In the TP53 mutant group, 67 samples $(76 \%)$ had one or more mutations in genes encoding chromatin remodeling proteins, including $A T R X, D N M T 3 A$, and $K M T 2 C$, which have been reported to be involved in cancer. This mutation co-occurrence may suggest a complex interplay in triple negative breast cancer. However, there was no association between TP53, PIK3CA or KMT2C mutation status and cancer staging. 


\section{Tumor Mutation Burden}

219 One of the biological hallmarks in cancer is genome instability. Genome alterations, which either

220 involve in carcinogenesis or occur as a result of widespread genome instability, can create

221 neoantigens and trigger immune response. We used the variant data to calculate the tumor

222 mutation burden for each sample and employed the same filtering scheme accounted for all

223 variants including both nonsynonymous and synonymous calls. Tumor mutation burden was

224 defined as the number of somatic base substitutions, and indels per megabase of coding genome

225 sequence examined. Synonymous mutations are counted in order to reduce sampling noise.

226 Though majority of synonymous mutations are not likely to cause tumor immunogenicity, they

227 may reflect mutational processes that will also result in nonsynonymous mutations and

228 neoantigens elsewhere in the genome (Diederichs et al. 2016; Supek et al. 2014). We found that

229 Thai TNBC has an average tumor mutation 7.3 variants per megabase (95\% CI 6.9-7.6), which

230 consists of nonsynonymous mutation 3.9 variants per megabase (95\% CI 3.7-4.1) and

231 synonymous mutation 3.4 variants per megabase (95\% CI 3.3-3.6). This data showed

232 significantly higher tumor mutation burden than median value (3.6 variants per megabase) found

233 in invasive ductal breast carcinoma cohort (Chalmers et al. 2017).

\section{Discussion}

235 Triple negative breast cancer (TNBC) is a heterogeneous disease with marked variation in

236 clinical characteristics and response to treatment (Blows et al. 2010). Genome data from

237 previous studies confirmed spectrum of mutational profiles in TNBC are diverse between each

238 patients and cohorts (Network 2012; Pereira et al. 2016; Shah et al. 2012; Stephens et al. 2012).

239 Though there are several explanations for diverse genome landscape in TNBC, patient's

240 ethnicity could play a significant role in this discrepancy. For the first time, this study provided 
241 the mutation profile of TNBC from Thai breast cancer patients. The data from our cohort showed

242 that, besides TP53 which is the most frequently mutated gene in TNBC, Thai TNBC patients

243 have much higher mutation frequencies in many cancer genes than Western patients. This

244 increase could be due to its exceptionally different pattern of somatic genome alterations in Thai

245 TNBC or its representation of higher tumor mutation burden that occurs extensively throughout

246 cancer genome or both.

247 TP53 remains the most commonly mutated gene in Thai TNBC similar to other reported studies

248 (Lefebvre et al. 2016; Network 2012; Pereira et al. 2016). This gene is widely considered

249 guardian of the genome due to its crucial function in maintaining genome integrity, regulating

250 cell cycle and initiating apoptosis. Numerous types of mutations in TP53 are found in various

251 cancers and mutations occur throughout the entire TP53 as expected in loss-of-function (LOF)

252 mutations in tumor suppressor gene. We also identify several recurring mutations in TP53

253 including R175H, Y220C, R248Q, and R273H, which together account for $26 \%$ of TP53 mutant

254 group. These 4 mutations are hotspot mutations that believed to be oncogenic missense variants

255 (Soussi \& Wiman 2015). Previous study in breast cancer showed that oncogenic TP53 variants in

256 DNA binding domain (amino acid position 102-292) are associated with reduced survival

257 compared to wild-type TP53 with an exception of Y220C, which is associated with better

258 survival in breast cancer (Olivier et al. 2006). However, such association could not be identified

259 likely due to very few mortality during our 3-year follow-up period.

PIK3CA mutations are usually enriched in hormonal receptor positive tumor at $29-45 \%$, with

261 lower frequency in TNBC (Network 2012). PIK3CA is the most commonly mutated oncogene in

262 Thai TNBC cohort similar to other breast cancer studies. Nevertheless, the mutation frequency of

263 PIK3CA in Thai TNBC is much higher than previously published data (Network 2012; Pereira et 
264 al. 2016; Shah et al. 2012). H1074R is the major PIK3CA hotspot mutation followed by K733R

265 and E545K. These variants are known oncogenic gain-of-function mutations found in multiple

266 types of cancers. Together with other PI3K pathway members, TNBC harboring mutations in

267 PIK3CA and its related genes could be potential targets for PI3K inhibitors.

$268 B R C A 1$ and $B R C A 2$ encode proteins that become parts of a complex that repairs double-strand

269 DNA breaks. They are critical for maintaining genome integrity. Breast cancers occurred in most

270 germline BRCA1 mutation carriers are TNBC. By contrast, there is no characteristic breast

271 cancer subtype in $B R C A 2$ carriers (Atchley et al. 2008). Most TNBC patients do not harbor

272 germline $B R C A 1$ or $B R C A 2$ mutations. However, pathological high-grade breast cancers and

273 TNBC often showed somatic mutations or abnormal expression of $B R C A 1$ or $B R C A 2$ (von

274 Wahlde et al. 2017). Thai TNBC displayed high prevalence of somatic alterations in both genes.

275 By comparison with 3 Western cohorts, the difference was less obvious because the mutation

276 frequencies did vary from one cohort to another. Nevertheless, defects in genome repair

277 machinery related to $B R C A 1$ and $B R C A 2$ mutations could make treatment with platinum-based

278 chemotherapy or PARP inhibitors more effective in those patients (Papadimitriou et al. 2018;

279 Vollebergh et al. 2014).

280 The KMT2C is noticeable because it is the second most commonly mutated gene (58\%) in our

281 Thai TNBC cohort. KMT2C is a gene in myeloid/lymphoid or mixed-lineage leukemia (MLL)

282 family and encodes MLL3 lysine-specific histone methyltransferase enzyme. H3 'Lys-4'

283 methylation in histone by the enzyme represents a specific tag for epigenetic transcriptional

284 activation (Rao \& Dou 2015). LOF mutations in KMT2C are found in myeloid leukemia,

285 melanoma, glioblastoma multiforme, hepatocellular carcinoma, esophageal cancer, colorectal

286 cancer and pancreatic cancer (Fujimoto et al. 2012; Garcia-Sanz et al. 2017; Liu et al. 2015; Lu 
287 et al. 2016; Song et al. 2014). Though the biological role of MLL3 histone methyltransferase in

288 carcinogenesis remains unknown, LOF mutations and downregulation of this gene in cancers

289 suggest that KMT2C may act as tumor suppressor gene (Xia et al. 2015). KMT2C mutations are

290 also reported in TNBC (Liu et al. 2015). However, Thai TNBC demonstrates substantially higher

291 prevalence of $K M T 2 C$ mutations than TNBC data from Caucasian population (Network 2012;

292 Pereira et al. 2016). A study from African American TNBC also displayed higher KMT2C

293 mutations than Caucasian patients (Ademuyiwa et al. 2017). A recently published data identified

$294 K M T 2 C$ mutations in 21\% of breast cancer patients from Singapore and Korea (Yap et al. 2018).

295 Interestingly, KMT2C has one identified hotspot variant; S338L (31\%) which has been

296 previously reported in colorectal cancer (Lu et al. 2016). This observation suggests that

297 epigenetic change may contribute to the development of TNBC and play significant role in Thai

298 TNBC patients. This data could lead to a new insight on epigenetic role of breast carcinogenesis.

299 Further investigation is warranted to provide better understanding on mechanisms of $K M T 2 C$ and 300 a novel treatment strategy.

301 As previously mentioned, Thai TNBC has generally higher mutation burden than breast cancer in 302 Western patients. The reason of this finding remains not fully explained. Previous study showed 303 that a subset of TNBC harbors somatic mutations in genome repair system (Shah et al. 2012; 304 Stephens et al. 2012). Higher tumor mutational load was observed in hormonal receptor negative 305 than hormonal receptor positive breast cancer (Barrett et al. 2018; Haricharan et al. 2014). Early 306 phase of anti-PD-1 clinical trials also showed higher response rate in TNBC than hormonal 307 receptor positive breast cancer (Nanda et al. 2016; Rugo et al. 2018). Our finding suggests that 308 immunotherapy could provide benefit to some Thai TNBC patients. 
309 In this study, the data from Thai TNBC would contribute much-needed information from Asian

310 patients to breast cancer genome landscape and provide another evidence on role of KMT2C in

311 breast carcinogenesis. However, our study has 3 major limitations. First, only patients from

312 Siriraj and King Chulalongkorn Memorial Hospitals were recruited. Both Bangkok-based

313 university hospitals were the largest hospitals in Thailand and served as major referral centers of

314 Thailand's healthcare system. Though breast cancer patients treated at our hospitals came from

315 all over the country, many patients from other regions of Thailand who could be treated locally

316 did not participate in the study. The data may not represent the whole picture of Thai TNBC

317 patients. Second, the follow-up period up to 3 years was not long enough to observe clinically

318 meaningful association between genomic alterations and clinical outcomes. Further study would

319 be required. Third, genome sequencing was done on only tumor gDNA extracted from FFPE

320 samples. It has been recognized that the quality of gDNA from FFPE is lower than fresh samples

321 and potentially causes variant call discrepancy. Our study chose to focus on list of cancer-

322 associated genes and apply variant call only when genomic regions have sufficient sequencing

323 depth. This approach has shown to minimize erroneous variant calls, improve precision and

324 acceptable correlation with matched normal-tumor pair sequencing (Chalmers et al. 2017; Oh et

325 al. 2015; Teer et al. 2017). Nevertheless, comparison of our TMB data with previously published

326 studies could be limited by differences in study designs and data analysis methods.

\section{Conclusion}

328 In conclusion, this study is the first cohort of Thai TNBC patients that demonstrated a distinctive

329 genome alterations including higher mutational burden, higher mutation frequencies on several

330 cancer-associated genes and mutations in $K M T 2 C$. These results support the genomic

331 heterogeneity between Caucasian and Thai TNBC and could present the new therapeutic 
332 approach on histone modification and immunotherapy in TNBC patients. Further investigation is

333 warranted to provide better understanding on role of KMT2C in breast carcinogenesis.

\section{Acknowledgement}

335 We wish to thank all participants for their cooperation and contribution to our study. We thank

336 all physicians and health professionals for their patient's clinical care. We also thank the

337 Research University Network (RUN) - Thailand for the administrative support.

338

339

340

341

342

343

344

345

346

347

348

349

350

351

352

\section{References}

1. Ademuyiwa FO, Tao Y, Luo J, Weilbaecher K, and Ma CX. 2017. Differences in the mutational landscape of triple-negative breast cancer in African Americans and Caucasians. Breast Cancer Res Treat 161:491-499. 10.1007/s10549-016-4062-y

2. Atchley DP, Albarracin CT, Lopez A, Valero V, Amos CI, Gonzalez-Angulo AM, Hortobagyi GN, and Arun BK. 2008. Clinical and pathologic characteristics of patients with BRCA-positive and BRCA-negative breast cancer. J Clin Oncol 26:4282-4288. $10.1200 /$ jco.2008.16.6231

3. Banegas MP, and Li CI. 2012. Breast cancer characteristics and outcomes among Hispanic Black and Hispanic White women. Breast Cancer Res Treat 134:1297-1304.

$10.1007 / \mathrm{s} 10549-012-2142-1$

4. Barrett MT, Lenkiewicz E, Malasi S, Basu A, Yearley JH, Annamalai L, McCullough AE, Kosiorek HE, Narang P, Wilson Sayres MA, Chen M, Anderson KS, and Pockaj BA. 2018. The association of genomic lesions and PD-1/PD-L1 expression in resected triple-negative breast cancers. Breast Cancer Research 20:71. 10.1186/s13058-018-1004-0 
353 5. Blows FM, Driver KE, Schmidt MK, Broeks A, van Leeuwen FE, Wesseling J, Cheang MC,

354 Gelmon K, Nielsen TO, Blomqvist C, Heikkila P, Heikkinen T, Nevanlinna H, Akslen LA,

355 Begin LR, Foulkes WD, Couch FJ, Wang X, Cafourek V, Olson JE, Baglietto L, Giles GG,

356 Severi G, McLean CA, Southey MC, Rakha E, Green AR, Ellis IO, Sherman ME,

357 Lissowska J, Anderson WF, Cox A, Cross SS, Reed MW, Provenzano E, Dawson SJ,

358 Dunning AM, Humphreys M, Easton DF, Garcia-Closas M, Caldas C, Pharoah PD, and

359 Huntsman D. 2010. Subtyping of breast cancer by immunohistochemistry to investigate a

360 relationship between subtype and short and long term survival: a collaborative analysis of

361 data for 10,159 cases from 12 studies. PLoS Med 7:e1000279.

362

363

364

6. Chalmers ZR, Connelly CF, Fabrizio D, Gay L, Ali SM, Ennis R, Schrock A, Campbell B, 10.1371/journal.pmed.1000279

7. Chen L, and Li CI. 2015. Racial disparities in breast cancer diagnosis and treatment by Shlien A, Chmielecki J, Huang F, He Y, Sun J, Tabori U, Kennedy M, Lieber DS, Roels S, White J, Otto GA, Ross JS, Garraway L, Miller VA, Stephens PJ, and Frampton GM. 2017. Analysis of 100,000 human cancer genomes reveals the landscape of tumor mutational burden. Genome Med 9:34. 10.1186/s13073-017-0424-2 D, Karschnia P, Linsenmeier M, Maulhardt T, Mohrmann L, Morstein J, Paffenholz SV, Ropenack P, Ruckert T, Sandig L, Schell M, Steinmann A, Voss G, Wasmuth J, Weinberger ME, and Wullenkord R. 2016. The dark matter of the cancer genome: aberrations in 
375 regulatory elements, untranslated regions, splice sites, non-coding RNA and synonymous

376 mutations. EMBO Mol Med 8:442-457. 10.15252/emmm.201506055

377

378

379

9. Fujimoto A, Totoki Y, Abe T, Boroevich KA, Hosoda F, Nguyen HH, Aoki M, Hosono N, Kubo M, Miya F, Arai Y, Takahashi H, Shirakihara T, Nagasaki M, Shibuya T, Nakano K, Watanabe-Makino K, Tanaka H, Nakamura H, Kusuda J, Ojima H, Shimada K, Okusaka T, Ueno M, Shigekawa Y, Kawakami Y, Arihiro K, Ohdan H, Gotoh K, Ishikawa O, Ariizumi S, Yamamoto M, Yamada T, Chayama K, Kosuge T, Yamaue H, Kamatani N, Miyano S, Nakagama H, Nakamura Y, Tsunoda T, Shibata T, and Nakagawa H. 2012. Whole-genome sequencing of liver cancers identifies etiological influences on mutation patterns and recurrent mutations in chromatin regulators. Nat Genet 44:760-764. 10.1038/ng.2291

10. Gao J, Aksoy BA, Dogrusoz U, Dresdner G, Gross B, Sumer SO, Sun Y, Jacobsen A, Sinha R, Larsson E, Cerami E, Sander C, and Schultz N. 2013. Integrative analysis of complex cancer genomics and clinical profiles using the cBioPortal. Sci Signal 6:pl1.

\subsection{6/scisignal.2004088}

11. Garcia-Sanz P, Trivino JC, Mota A, Perez Lopez M, Colas E, Rojo-Sebastian A, Garcia A, Gatius S, Ruiz M, Prat J, Lopez-Lopez R, Abal M, Gil-Moreno A, Reventos J, Matias-Guiu X, and Moreno-Bueno G. 2017. Chromatin remodelling and DNA repair genes are frequently mutated in endometrioid endometrial carcinoma. Int J Cancer 140:1551-1563. 10.1002/ijc.30573

12. Haricharan S, Bainbridge MN, Scheet P, and Brown PH. 2014. Somatic mutation load of estrogen receptor-positive breast tumors predicts overall survival: an analysis of genome sequence data. Breast Cancer Res Treat 146:211-220. 10.1007/s10549-014-2991-x 
397

398

399

400

401

402

403

404

405

406

407

408

409

410

411

412

413

414

415

416

417

418

13. Huo D, Hu H, Rhie SK, Gamazon ER, Cherniack AD, Liu J, Yoshimatsu TF, Pitt JJ, Hoadley KA, Troester M, Ru Y, Lichtenberg T, Sturtz LA, Shelley CS, Benz CC, Mills GB, Laird PW, Shriver CD, Perou CM, and Olopade OI. 2017. Comparison of Breast Cancer Molecular Features and Survival by African and European Ancestry in The Cancer Genome Atlas. JAMA Oncol 3:1654-1662. 10.1001/jamaoncol.2017.0595

14. Lefebvre C, Bachelot T, Filleron T, Pedrero M, Campone M, Soria JC, Massard C, Levy C, Arnedos M, Lacroix-Triki M, Garrabey J, Boursin Y, Deloger M, Fu Y, Commo F, Scott V, Lacroix L, Dieci MV, Kamal M, Dieras V, Goncalves A, Ferrerro JM, Romieu G, Vanlemmens L, Mouret Reynier MA, Thery JC, Le Du F, Guiu S, Dalenc F, Clapisson G, Bonnefoi H, Jimenez M, Le Tourneau C, and Andre F. 2016. Mutational Profile of Metastatic Breast Cancers: A Retrospective Analysis. PLoS Med 13:e1002201. 10.1371/journal.pmed.1002201

15. Liu L, Kimball S, Liu H, Holowatyj A, and Yang ZQ. 2015. Genetic alterations of histone lysine methyltransferases and their significance in breast cancer. Oncotarget 6:2466-2482. 10.18632/oncotarget.2967

16. Lu YW, Zhang HF, Liang R, Xie ZR, Luo HY, Zeng YJ, Xu Y, Wang LM, Kong XY, and Wang KH. 2016. Colorectal Cancer Genetic Heterogeneity Delineated by Multi-Region Sequencing. PLoS One 11:e0152673. 10.1371/journal.pone.0152673

17. Malorni L, Shetty PB, De Angelis C, Hilsenbeck S, Rimawi MF, Elledge R, Osborne CK, De Placido S, and Arpino G. 2012. Clinical and biologic features of triple-negative breast cancers in a large cohort of patients with long-term follow-up. Breast Cancer Res Treat 136:795-804. 10.1007/s10549-012-2315-y 
419 18. McLaren W, Gil L, Hunt SE, Riat HS, Ritchie GRS, Thormann A, Flicek P, and

420 Cunningham F. 2016. The Ensembl Variant Effect Predictor. Genome Biology 17:122. $10.1186 / \mathrm{s} 13059-016-0974-4$

422

423

424

425

426

427

428

429

430

431

432

433

434

435

436

437

438

439

440

441

19. Nanda R, Chow LQ, Dees EC, Berger R, Gupta S, Geva R, Pusztai L, Pathiraja K, Aktan G, Cheng JD, Karantza V, and Buisseret L. 2016. Pembrolizumab in Patients With Advanced Triple-Negative Breast Cancer: Phase Ib KEYNOTE-012 Study. J Clin Oncol 34:24602467. $10.1200 /$ jco.2015.64.8931

20. Network CGA. 2012. Comprehensive molecular portraits of human breast tumours. Nature 490:61-70. 10.1038/nature11412

21. Oh E, Choi YL, Kwon MJ, Kim RN, Kim YJ, Song JY, Jung KS, and Shin YK. 2015. Comparison of Accuracy of Whole-Exome Sequencing with Formalin-Fixed ParaffinEmbedded and Fresh Frozen Tissue Samples. PLoS One 10:e144162.

$$
\text { 10.1371/journal.pone.0144162 }
$$

22. Olivier M, Langerod A, Carrieri P, Bergh J, Klaar S, Eyfjord J, Theillet C, Rodriguez C, Lidereau R, Bieche I, Varley J, Bignon Y, Uhrhammer N, Winqvist R, Jukkola-Vuorinen A, Niederacher D, Kato S, Ishioka C, Hainaut P, and Borresen-Dale AL. 2006. The clinical value of somatic TP53 gene mutations in 1,794 patients with breast cancer. Clin Cancer Res 12:1157-1167. 10.1158/1078-0432.CCR-05-1029

23. Papadimitriou M, Mountzios G, and Papadimitriou CA. 2018. The role of PARP inhibition in triple-negative breast cancer: Unraveling the wide spectrum of synthetic lethality. Cancer Treatment Reviews 67:34-44. 10.1016/j.ctrv.2018.04.010

24. Pereira B, Chin SF, Rueda OM, Vollan HK, Provenzano E, Bardwell HA, Pugh M, Jones L, Russell R, Sammut SJ, Tsui DW, Liu B, Dawson SJ, Abraham J, Northen H, Peden JF, 
442

443

444

445

446

447

448

449

450

451

452

453

454

455

456

457

458

459

460

461

462

463

Mukherjee A, Turashvili G, Green AR, McKinney S, Oloumi A, Shah S, Rosenfeld N, Murphy L, Bentley DR, Ellis IO, Purushotham A, Pinder SE, Borresen-Dale AL, Earl HM, Pharoah PD, Ross MT, Aparicio S, and Caldas C. 2016. The somatic mutation profiles of 2,433 breast cancers refines their genomic and transcriptomic landscapes. Nat Commun 7:11479. 10.1038/ncomms11479

25. Rao RC, and Dou Y. 2015. Hijacked in cancer: the KMT2 (MLL) family of methyltransferases. Nat Rev Cancer 15:334-346. 10.1038/nrc3929

26. Rhee J, Han S-W, Oh D-Y, Kim JH, Im S-A, Han W, Ae Park I, Noh D-Y, Bang Y-J, and Kim T-Y. 2008. The clinicopathologic characteristics and prognostic significance of triplenegativity in node-negative breast cancer. BMC Cancer 8:307. 10.1186/1471-2407-8-307

27. Rugo HS, Delord JP, Im SA, Ott PA, Piha-Paul SA, Bedard PL, Sachdev J, Tourneau CL, van Brummelen EMJ, Varga A, Salgado R, Loi S, Saraf S, Pietrangelo D, Karantza V, and Tan AR. 2018. Safety and Antitumor Activity of Pembrolizumab in Patients with Estrogen Receptor-Positive/Human Epidermal Growth Factor Receptor 2-Negative Advanced Breast Cancer. Clin Cancer Res 24:2804-2811. 10.1158/1078-0432.ccr-17-3452

28. Shah SP, Roth A, Goya R, Oloumi A, Ha G, Zhao Y, Turashvili G, Ding J, Tse K, Haffari G, Bashashati A, Prentice LM, Khattra J, Burleigh A, Yap D, Bernard V, McPherson A, Shumansky K, Crisan A, Giuliany R, Heravi-Moussavi A, Rosner J, Lai D, Birol I, Varhol R, Tam A, Dhalla N, Zeng T, Ma K, Chan SK, Griffith M, Moradian A, Cheng SW, Morin GB, Watson P, Gelmon K, Chia S, Chin SF, Curtis C, Rueda OM, Pharoah PD, Damaraju S, Mackey J, Hoon K, Harkins T, Tadigotla V, Sigaroudinia M, Gascard P, Tlsty T, Costello JF, Meyer IM, Eaves CJ, Wasserman WW, Jones S, Huntsman D, Hirst M, Caldas C, Marra 
464

465

466

467

468

469

470

471

472

473

474

475

476

477

478

479

480

481

482

483

484

485

486

MA, and Aparicio S. 2012. The clonal and mutational evolution spectrum of primary triplenegative breast cancers. Nature 486:395-399. 10.1038/nature10933

29. Song Y, Li L, Ou Y, Gao Z, Li E, Li X, Zhang W, Wang J, Xu L, Zhou Y, Ma X, Liu L, Zhao Z, Huang X, Fan J, Dong L, Chen G, Ma L, Yang J, Chen L, He M, Li M, Zhuang X,

Huang K, Qiu K, Yin G, Guo G, Feng Q, Chen P, Wu Z, Wu J, Ma L, Zhao J, Luo L, Fu M, Xu B, Chen B, Li Y, Tong T, Wang M, Liu Z, Lin D, Zhang X, Yang H, Wang J, and Zhan Q. 2014. Identification of genomic alterations in oesophageal squamous cell cancer. Nature 509:91-95. 10.1038/nature13176

30. Soussi T, and Wiman KG. 2015. TP53: an oncogene in disguise. Cell Death Differ 22:12391249. $10.1038 /$ cdd. 2015.53

31. Stephens PJ, Tarpey PS, Davies H, Van Loo P, Greenman C, Wedge DC, Nik-Zainal S, Martin S, Varela I, Bignell GR, Yates LR, Papaemmanuil E, Beare D, Butler A, Cheverton A, Gamble J, Hinton J, Jia M, Jayakumar A, Jones D, Latimer C, Lau KW, McLaren S, McBride DJ, Menzies A, Mudie L, Raine K, Rad R, Chapman MS, Teague J, Easton D, Langerod A, Oslo Breast Cancer C, Lee MT, Shen CY, Tee BT, Huimin BW, Broeks A, Vargas AC, Turashvili G, Martens J, Fatima A, Miron P, Chin SF, Thomas G, Boyault S, Mariani O, Lakhani SR, van de Vijver M, van 't Veer L, Foekens J, Desmedt C, Sotiriou C, Tutt A, Caldas C, Reis-Filho JS, Aparicio SA, Salomon AV, Borresen-Dale AL, Richardson AL, Campbell PJ, Futreal PA, and Stratton MR. 2012. The landscape of cancer genes and mutational processes in breast cancer. Nature 486:400-404. 10.1038/nature11017

32. Supek F, Minana B, Valcarcel J, Gabaldon T, and Lehner B. 2014. Synonymous mutations frequently act as driver mutations in human cancers. Cell 156:1324-1335.

10.1016/j.cell.2014.01.051 
487 33. Teer JK, Zhang Y, Chen L, Welsh EA, Cress WD, Eschrich SA, and Berglund AE. 2017.

488 Evaluating somatic tumor mutation detection without matched normal samples. Hum

Genomics 11:22. 10.1186/s40246-017-0118-2

490

491

492

493

494

495

496

497

498

499

500

501

502

503

504

505

506

507

508

509
34. Thike AA, Cheok PY, Jara-Lazaro AR, Tan B, Tan P, and Tan PH. 2009. Triple-negative breast cancer: clinicopathological characteristics and relationship with basal-like breast cancer. Modern Pathology 23:123. 10.1038/modpathol.2009.145

35. Vollebergh MA, Lips EH, Nederlof PM, Wessels LF, Wesseling J, Vd Vijver MJ, de Vries EG, van Tinteren H, Jonkers J, Hauptmann M, Rodenhuis S, and Linn SC. 2014. Genomic patterns resembling BRCA1 - and BRCA2-mutated breast cancers predict benefit of intensified carboplatin-based chemotherapy. Breast Cancer Res 16:R47. 10.1186/bcr3655

36. von Wahlde M-K, Timms KM, Chagpar A, Wali VB, Jiang T, Bossuyt V, Saglam O, Reid J, Gutin A, Neff C, Lanchbury JS, Hatzis C, Hofstatter E, and Pusztai L. 2017. Intratumor Heterogeneity of Homologous Recombination Deficiency in Primary Breast Cancer. Clinical Cancer Research 23:1193.

37. Xia M, Xu L, Leng Y, Gao F, Xia H, Zhang D, and Ding X. 2015. Downregulation of MLL3 in esophageal squamous cell carcinoma is required for the growth and metastasis of cancer cells. Tumour Biol 36:605-613. 10.1007/s13277-014-2616-3

38. Yap Y-S, Singh AP, Lim JHC, Ahn J-H, Jung K-H, Kim J, Dent RA, Ng RCH, Kim S-B, and Chiang DY. 2018. Elucidating therapeutic molecular targets in premenopausal Asian women with recurrent breast cancers. npj Breast Cancer 4:19. 10.1038/s41523-018-0070-x 


\section{Abbreviation}

511 BMI Body mass index

512 CADD Combined Annotation Dependent Depletion

513 COSMIC Catalogue of Somatic Mutations in Cancer

514 DNA Deoxyribonucleic acid

515 ER Estrogen receptor

516 ExAC Exome Aggregation Database

517 FFPE Formalin-fixed, paraffin-embedded

518 gDNA Genomic deoxyribonucleic acid

519 HER2 Human epidermal growth factor receptor 2

520 HGMD Human Gene Mutation Database

521 LOF Loss of function

522 LRT Likelihood Ratio Test

523 OMIM Online Mendelian Inheritance in Men

524 PAM50 Prosigna Intrinsic Subtype of Breast Cancer

525 PARP Poly (ADP-ribose) polymerase

526 PI3K Phosphatidylinositide 3-kinases

527 PR Progesterone receptor 
528 SIFT Sorting Intolerance from Tolerance

529 SNP Single nucleotide polymorphism

530 SO Sequence Ontology

531 TCGA The Cancer Genome Atlas

532 TNBC Triple negative breast cancer

533 UTR Untranslated region 


\section{Table 1 (on next page)}

Frequencies of ten most commonly mutated genes in Thai TNBC compared to other cohorts.

$\mathrm{n}$ indicated number of patients in each cohort 


\begin{tabular}{|llllll|}
\hline Gene & $\begin{array}{l}\text { Thai } \\
\text { TNBC }\end{array}$ & TCGA & METABRIC & French & $\begin{array}{l}\text { Pearson } \\
\text { Chi Square }\end{array}$ \\
& $\begin{array}{lllll}(\mathbf{n}=\mathbf{1 1 6}) \\
\text { TP53 }\end{array}$ & $\mathbf{( n = 9 3 )}$ & $\mathbf{( n = 2 4 5 )}$ & $\mathbf{( n = 5 1 )}$ & p-value \\
KMT2C & $57.76 \%$ & $82.80 \%$ & $80.00 \%$ & $74.51 \%$ & 0.519 \\
SYNE1 & $31.71 \%$ & $5.38 \%$ & $8.16 \%$ & $1.96 \%$ & $<0.001$ \\
PIK3CA & $23.28 \%$ & $8.60 \%$ & $16.73 \%$ & $5.88 \%$ & $<0.001$ \\
BRCA1 & $21.55 \%$ & $15.05 \%$ & $4.49 \%$ & $11.76 \%$ & 0.027 \\
& & & & $1.96 \%$ & $<0.001$ \\
BRCA2 & $18.10 \%$ & $6.45 \%$ & $1.63 \%$ & $3.92 \%$ & $<0.001$ \\
NF1 & $14.66 \%$ & $2.15 \%$ & $3.27 \%$ & $3.92 \%$ & $<0.001$ \\
PTEN & $11.21 \%$ & $1.08 \%$ & $6.53 \%$ & $5.88 \%$ & 0.033 \\
RB1 & $10.34 \%$ & $4.30 \%$ & $4.90 \%$ & $5.88 \%$ & 0.19 \\
ARID1B & $6.90 \%$ & $4.30 \%$ & $4.08 \%$ & $1.96 \%$ & 0.495 \\
\hline
\end{tabular}

1 Table 1 - Frequencies of ten most commonly mutated genes in Thai TNBC compared to other

2 cohorts. (n indicated number of patients in each cohort) 
Table 2 (on next page)

Frequencies of mutated genes among 3 published cohorts

$\mathrm{n}$ indicated number of patients in each cohort 


\begin{tabular}{lrrrr} 
Gene & $\begin{array}{l}\text { TCGA } \\
(\mathbf{n = 9 3 )}\end{array}$ & $\begin{array}{l}\text { METABRIC } \\
(\mathbf{n = 2 4 5})\end{array}$ & $\begin{array}{l}\text { French } \\
(\mathbf{n = 5 1})\end{array}$ & \multicolumn{2}{c}{$\begin{array}{l}\text { Pearson } \\
\text { phi Square }\end{array}$} \\
TP53 & $82.80 \%$ & $80.00 \%$ & $74.51 \%$ & 0.494 \\
KMT2C & $6.45 \%$ & $8.16 \%$ & $1.96 \%$ & 0.278 \\
SYNE1 & $5.38 \%$ & $16.73 \%$ & $5.88 \%$ & 0.006 \\
PIK3CA & $8.60 \%$ & $18.37 \%$ & $11.76 \%$ & 0.063 \\
& & & & \\
BRCA1 & $15.05 \%$ & $4.49 \%$ & $1.96 \%$ & 0.001 \\
BRCA2 & $6.45 \%$ & $1.63 \%$ & $3.92 \%$ & 0.068 \\
NF1 & $2.15 \%$ & $3.27 \%$ & $3.92 \%$ & 0.812 \\
PTEN & $1.08 \%$ & $6.53 \%$ & $5.88 \%$ & 0.123 \\
RB1 & $4.30 \%$ & $4.90 \%$ & $5.88 \%$ & 0.915 \\
ARID1B & $4.30 \%$ & $4.08 \%$ & $1.96 \%$ & 0.749
\end{tabular}

1 Table 2 - Frequencies of mutated genes among 3 published cohorts ( $\mathrm{n}$ indicated number of 2 patients in each cohort) 
Figure 1

Somatic Mutation Frequencies among 4 TNBC cohorts. Bar chart showed 10 most commonly mutated in Thai TNBC comparing to TCGA, METABRIC and French cohorts.

* indicated the difference was statistically significant

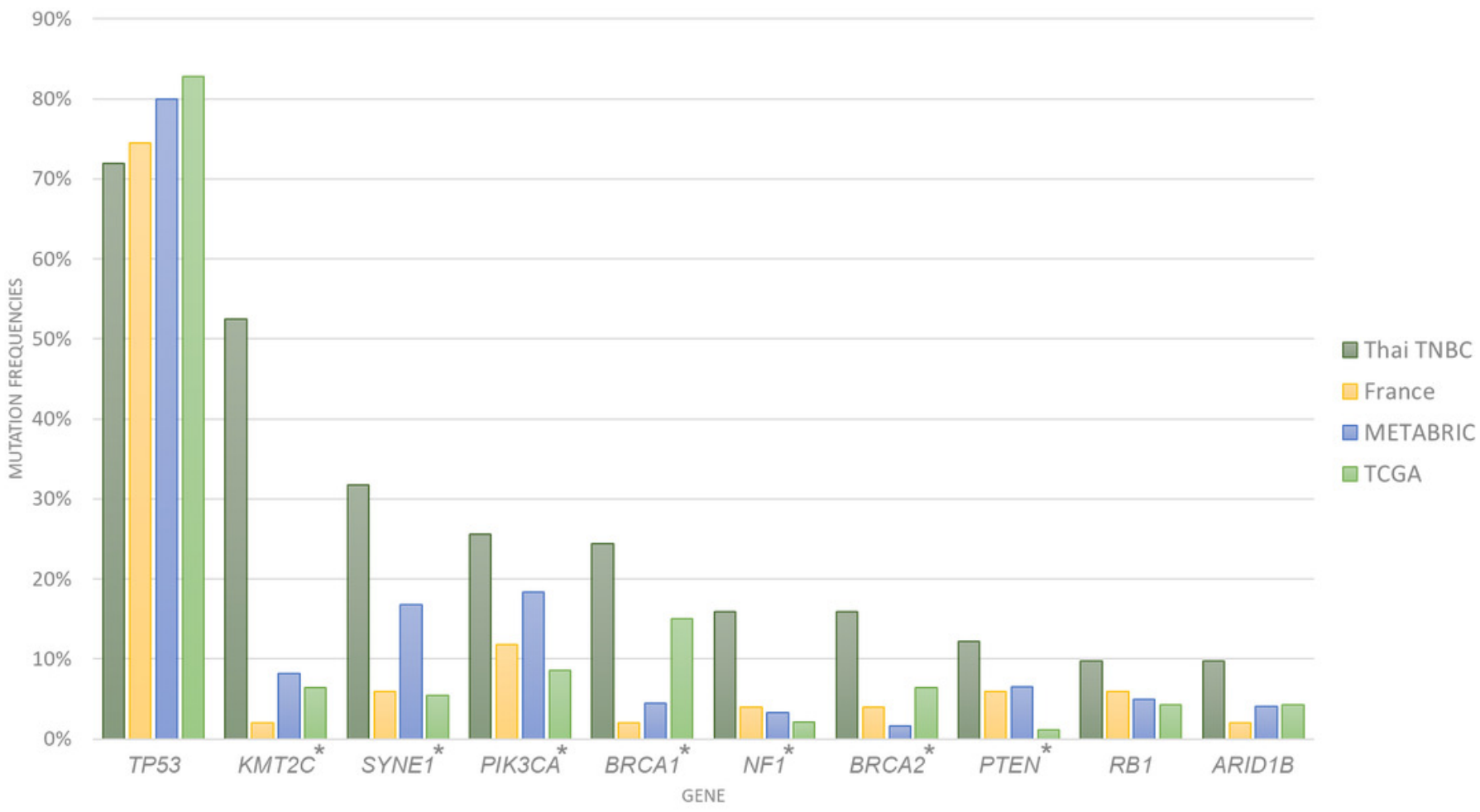




\section{Figure 2}

Landscape of co-occurrence and mutual exclusion of cancer gene mutations in Thai TNBC

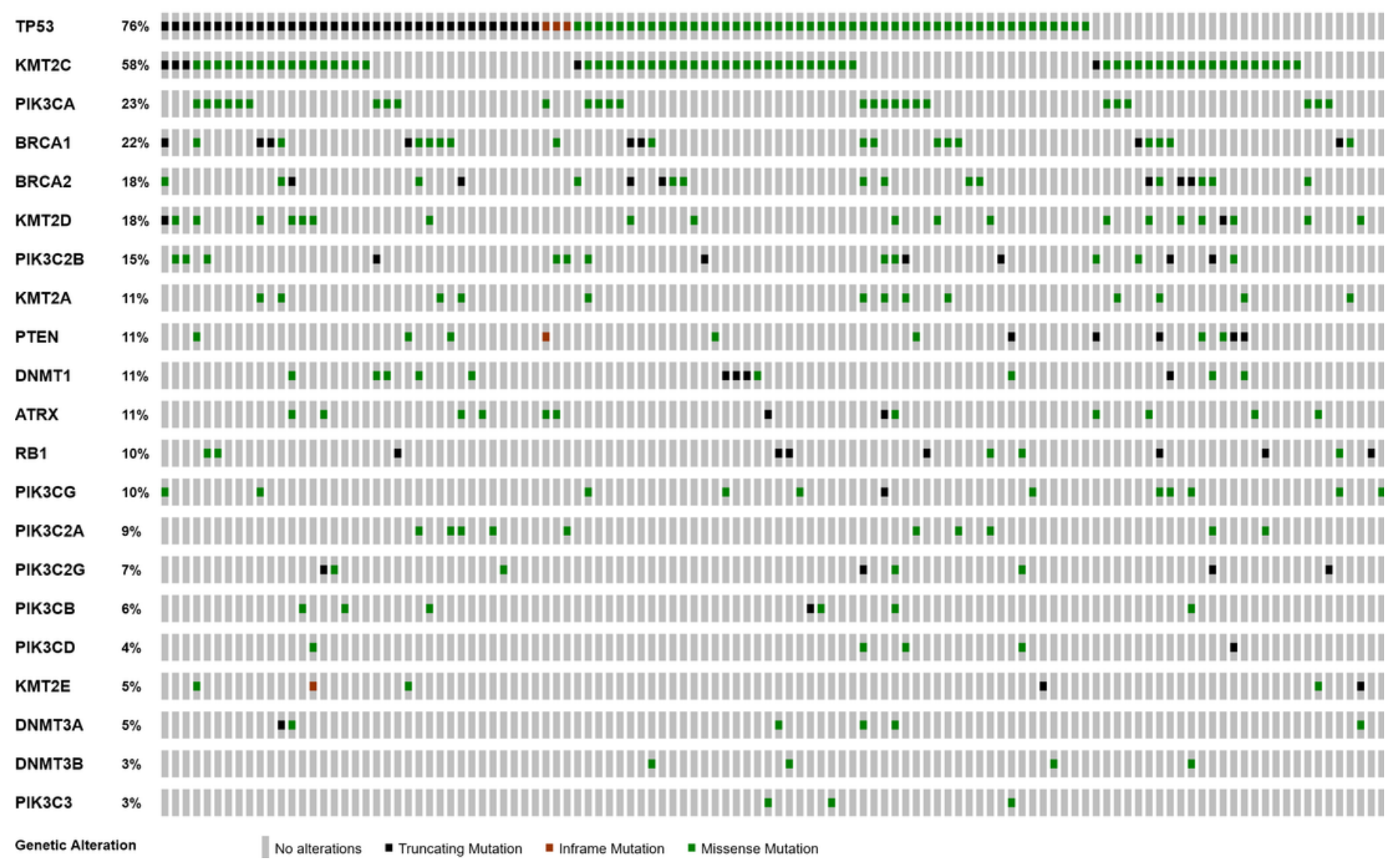




\section{Figure 3}

Mutational Spectrum of TP53, KMT2C and PIK3CA in TNBC. The images showed protein domains and the positions of specific mutations with most common type of mutations in each genes labelled.

A block dot indicated a truncating mutation; a green dot indicated a missense mutation; and a purple dot indicated other types of mutation. 

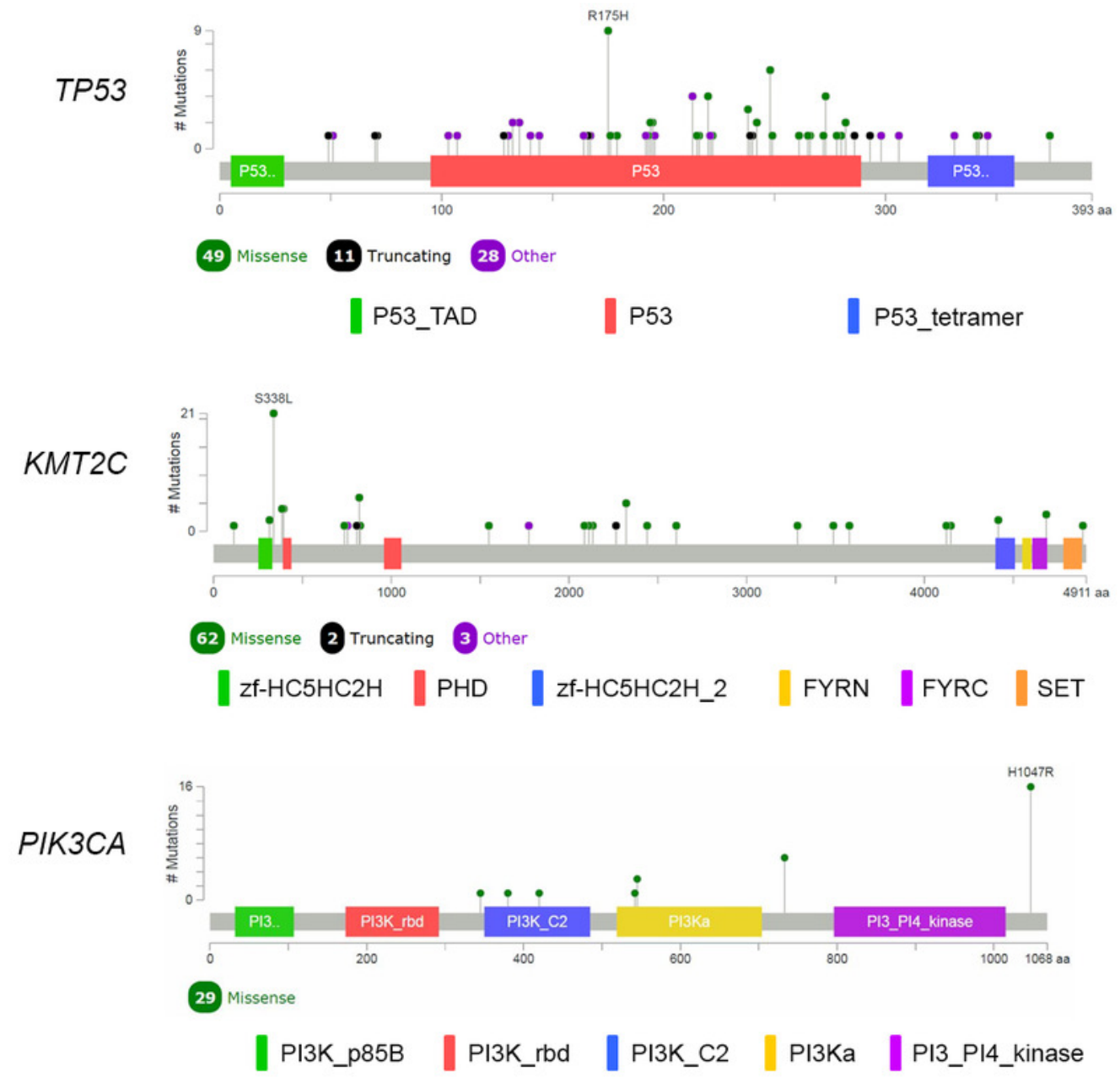